\title{
Rise of the Citizen Developer
}

\author{
By \\ Mary Lebens, Metropolitan State University \\ Roger Finnegan, Metropolitan State University \\ Steve Sorsen, Metropolitan State University \\ Jinal Shah, Metropolitan State University
}

G artner (Pham, 2019) reported that the developer talent shortage is the number one risk to organizations globally. Forbes (2021) reports that large tech companies are better able to acquire the talent that they need while smaller companies have fewer chances. This worldwide shortage of developers has made low- and no-code platforms important and necessary, particularly to smaller organizations. This paper investigates the use of these platforms in organizations, along with the role of workforce automation tools. A survey was conducted to find out how prevalent low- and no-code platforms and workforce automation tools are within companies. These platforms are used by citizen developers, employees who are working outside of the Information Technology (IT) department and are not professional programmers. With low-and no-code platforms citizen developers can create the applications that are needed by their work units or even their entire organizations. These platforms are seen as key to the demands of digital transformation. The results of this study are that companies both large and small are making use of lowand no-code platforms, as well as workforce automation tools. In addition, the majority of organizations have em-
This research examines the use of low- and no-code development platforms to increase the development capabilities of an organization. The research found that companies of all sizes are making use of these platforms, often in conjunction with workflow automation. ployees outside of the IT department who are creating technology solutions. The broad implication of this research is that citizen developers using lowand no-code platforms to create technology solutions may be the solution to the current shortage of developers.

By using low- and no-code platforms, the citizen developer can create the applications that the manager needs for their team. This increases the technology available to the organization while at the same time reducing the pressure on the IT department.

Keywords: Low-code, no-code, citizen developer, digital transformation

Copyright 2021, Mary Lebens, Roger Finnegan, Steve Sorsen, Jinal Shah. This article is published under a Creative Commons BY-NC license. Permission is granted to copy and distribute this article for non-commercial purposes, in both printed and electronic formats. 
There is currently a shortage of Information Technology (IT) software developers, at the same time that outsourcing costs are rising (Bowman, 2018). Thus, IT departments are having difficulty keeping up with the demands of business users (Tuomi, 2018). Due to the shortage of IT talent, many corporate users are waiting long periods of time to have their business needs addressed. At the same time no-code development platforms have recently become available. This has provided individuals without computer coding skills the opportunity to develop applications, resulting in the rise of the citizen developer. Gartner defines the citizen developer as:

"[A] user who creates new business applications for consumption by others using development and runtime environments sanctioned by corporate IT. End users can build departmental, enterprise and even public applications using shared services, fourth-generation language development platforms and cloud computing services (Gartner Information Technology Glossary, 2021).

This research study delves into the rise of the citizen developer by examining if organizations are making use of low- and no-code platforms and workflow automation tools. The research examines this question from the viewpoint of both developers and non-developers. As noted, there is currently a shortage of software developers worldwide (Bowman, 2018; Muraski et al., 2021; Tuomi, 2018). This is causing issues for companies as they try to develop applications for their internal and external use. The advent of the low- and no-code platforms provides a way to relieve this shortage. Developers can make use of low-code platforms to create applications more quickly than with conventional platforms, while at the same time business users can make use of no-code platforms to create the apps that they need to enhance business processes (Sahinaslan et al., 2021).

\section{Review of Research}

\section{Citizen Developers}

The organization IT department's role of implementing applications, analyzing data, refining performance, and presenting output is being challenged by cloud-based solutions and mobile platforms (Woo, 2020). To exacerbate this challenge, the growing demand for experienced IT professionals is increasing while the supply is dwindling (Tuomi, 2018). Since 2004 , the number of computer science and information systems graduates in the United States has been shrinking, while the Bureau of Labor Statistics predicts the hiring demand for computer-related careers to increase over 13\% from 2014 to 2024 (Alexander et al., 2011; Bowman, 2018). The IT field is predicted to have the third-fastest growth from 2019 to 2029, after healthcare and social support careers (Muraski et al., 2021). The decline in the number of IT gradu- ates in the United States began with the Dotcom bust of the early 2000s. Graduates with IT degrees fell from $5.1 \%$ in 2003 to $3.7 \%$ in 2010 . The number began to rise during the Great Recession but has never reached the 2003 levels again (Martinez \& Pearson, n.d.). In addition, Forbes states that the institutions of higher learning are not graduating students with the skills that organizations need (Pham, 2021). This means that companies are competing for the students that have the needed skills (Pham, 2021).

The citizen developer low- and no-code movement addresses this demand and puts emphasis on the end-user providing their own solutions (Fryling, 2019). Low- and no-code development is providing employees, who are not members of the IT department, the ability to create business applications to be used by the organization (Coleman, 2020). These employees are called "citizen developers" (Ng'ambi, 2020). Citizen developers use powerful toolsets to build modular applications, reducing the demand for IT professionals while driving efficiency and productivity (Totterdale, 2018). Citizen developers are problem solvers leveraging technology to create solutions (Ngambi, 2020). Using low- and no-code tools, citizen developers quickly create applications without formal training in programming (Lodge et al., 2018; Oltrogge et al., 2018).

For the business manager, the possibility of using low- and no-code platforms as well as workflow automation is a significant consideration. Gartner and Forrester have documented the increased use of low and no-code platforms, as well as the increasing technical capabilities of these platforms (Costello \& Rimhol, 2021; Rymer \& Koplowitz, 2019). With the expanding power of the platforms combined with the growing ranks of citizen developers, it makes sense for organizations to move in the direction of low-code and no-code platforms (Fryling, 2019). In most organizations the business units must wait to have their systems or processes enhanced since the IT departments have limited resources or are not able to acquire the resources that they need to complete the development (Bowman, 2018). Cultivating citizen developers allows business units to create their needed applications, and there is research showing that business employees are willing to use low- and no-code platforms (Ploder et al., 2019). These business users can self-serve their IT needs to at least some degree. While there is a possibility allowing business users to serve their own needs may create a conflict with the IT department, because IT employees may fear losing control or may resist for reasons of job protection (Van Looy, 2020). However, if end-user development is properly managed, particularly in regards to security, end-user development can provide a win-win situation for both departments (Alamin et al., 2021). Besides issues with 
security conflicts can arise between citizen developers and the IT department due to control over data, particularly sensitive data, as well as the use of unmanaged devices especially from remote locations (Matteson, 2020). Low- and no-code development, coupled with workflow automation, allows business units to build the applications that they need while the IT department concentrates on the strategic needs of the organization (Olariu et al., 2016).

\section{Low- and No-Code Development}

Low- and no-code development uses drag-anddrop technology to arrange predefined modules into modular applications (Hyun, 2019). Modular applications are extremely flexible, easy to create, and simple to update by moving pre-coded application modules in and out of the application (Hyun, 2019). The ease of use allows experienced users to develop applications without engaging enterprise IT, allowing users to move quickly and efficiently (Silva et al., 2020; Wild, 2021; Wolff, 2019). The user inputs their business requirements, making real-time changes while developing the app (Woo, 2020). Due to the ease of developing applications using low-and nocode platforms, Gartner predicts the market for lowcode development to grow 23\% in 2021 (Costello \& Rimhol, 2021). A 2020 study that found a majority of low- and no-code developers were working in startups or were self-employed, indicating these tools are most popular in small organizations (Gadberry \& Romero, 2020).

\section{Workflow Automation Tools}

The existing research shows that organizations which are using low- and no-code platforms are using workflow automation tools as well, since these tools are designed to complement each other (Rymer \& Koplowitz, 2019; Sahay et al., 2020). Workflow automation tools are usually sold alongside or integrated into low- and no-code development platforms, as is the case with Microsoft and Salesforce, which GlobalData ranked as leaders in both the workflow automation and the low-code/no-code space (Patterson, 2020). Microsoft's Power Automate tool enables the end user to build low-code/no-code workflows to automate tasks with Robotic Process Automation (RPA) (Steinmetz, 2021). Many workflow automation tools are packaged along with lowcode development tools, such as the SAP HXM suite and Microsoft Dynamics $365 \mathrm{HR}$, which are aimed at allowing Human Resources (HR) employees become citizen developers by automating business processes for the HR department (Shafagatova and Van Looy, 2021). Workflow automation tools compliment and facilitate the rise of the citizen developer by providing graphical user interfaces where users can drag and drop building blocks for their applications. This empowers users to create the business processes that they need without having the knowledge of a trained developer (O'Connor, 2021).

The SAP platform is another example of this integration between the low- and no-code platforms and workforce automation tools. SAP has three process automation tools; "Cloud Platform Workflow Management" for creating low-code enterprise workflows; "Ruum" for automating department processes; and "Intelligent Robotic Process Automation" for automating other business processes (Sayer, 2020). Other technology companies also have a simultaneous presence in workflow automation and low- and no-code development. Betty Blocks calls itself the first no-code platform for workflow automation, with a basic design that is easy to use (Vincent et al., 2020). Mendix, ServiceNow, and Appian are other tech companies considered as industry leaders by Gartner in the workflow automation space, while concurrently providing low- and no-code tools to complement workflow automation (Vincent et al., 2020).

\section{Digital Transformation}

Digital transformation is centered on adopting disruptive technologies into business operations to raise productivity and increase value (Anderson, 2020; Duarte \& Ebert, 2018). The goal of digital transformation is using transformative technology to increase productivity, improve efficiency of operations, and generate new revenue streams (Vial, 2019). One way organizations can meet this goal is by implementing Robotic Process Automation (RPA), which utilizes technology to digitize manual tasks and generate workflows to transition from process to process (Van der Aalst et al., 2018). RPA increases productivity and improves efficiency by automating rote tasks, freeing employees to spend more time on higher-level endeavors within the organization (Van der Aalst et al., 2018). RPA can be used to automate rule-based repetitive processes with clear inputs and outputs (Casey, 2020). In addition, RPA can be used to automate invoice processing, customer information validation, and employee onboarding $\mathrm{HR}$ (Browning, 2021). Low- and no-code platforms are integrated into RPA to provide the tools to automate tasks and workflows, making low- and no-code platforms critical to digital transformation as well (Sakhnyuk \& Sakhnyuk, 2020). Citizen developers will drive digital transformation by using low-and no-code platforms in conjunction with workflow automation, such as RPA (Sahay et al., 2020).

\section{Organizational Size}

Due to the very recent rise of low- and no-code development tools, as well as workflow automation, little research has been done on the size of the organizations using these tools (Moskal, 2021). Some experts speculate that these tools may give smaller 
organizations the opportunity to expand their application development, since the cost of using citizen developers is lower than the cost of hiring professional software developers (Tariq, 2021). A subset of low- and no-code development tools, such as Google App Cloud, Appian, and Zoho Creator, are specifically aimed at supporting small- to medium-scale organizations and are offered at a lower-price point to appeal to smaller organizations (Sahay et al., 2020). There are even some free low- and no-code platforms available to smaller organizations, such as Node-RED, which is a free open-source no-code platform that allows end users to create workflows using a drag-and-drop interface that runs in the browser (O'Leary \& Conway-Jones, 2020).

Preliminary research on low- and no-code development platforms shows an initial trend towards smaller organizations making use of these tools to expand application development. A study of small to medium size manufacturing companies found that workflow automation and low-code applications were effective for monitoring product production, indicating that these solutions could be helpful for smaller organizations (Hawkridge et al., 2021). In 2020, Bubble, Inc. surveyed 741 developers using no- and low-code platforms and found 59\% of them were self-employed or working for a start-up and another $17 \%$ were working in a small business (Gadberry \& Romero, 2020). Only 6\% of the developers surveyed indicated there were working in an enterprise organization (Gadberry \& Romero, 2020). Although the research on the size of organizations using low- and no-code platforms and workflow automation is in a very preliminary stage, the research points to these tools being most popular in smaller organizations.

\section{Research Questions}

Based on the review of previous research, the authors developed the following research questions:

- RQ1: What is the prevalence of low- and nocode platforms in organizations?

- RQ2: How does the use of low- and no-code platforms differ across sizes of organizations?

- RQ3: What is the prevalence of citizen developers in organizations?

- RQ4: What is the relationship between the use of low- and no-code platforms and workflow automation?

\section{The Protocol}

\section{Design of Survey Instrument}

The research team developed the survey questions based on the review of the existing literature in order to extend the research on the use of low- and no-code development environments, as well as the use of workflow automation, across organizations of different sizes. The survey instrument was designed using the Qualtrics XM online survey platform. The survey was validated using Qualtrics' Expert Review validation tool based on twelve industry-standard criteria, including accessibility, mobile support, and clarity. The survey length was purposefully short to encourage participation, containing seven research questions and one demographic question. Due to the difficulty that the team encountered in receiving completed surveys a convenience methodology was adapted.

\section{Description of Respondents}

The respondents were Information Technology (IT) professionals recruited through LinkedIn, Twitter, and the New Directions in IT Conference for a total of 1,536 potential respondents. The survey garnered 42 responses, for a response rate of 3\%. Thirty-eight of the responses were used in the analysis. The survey was conducted anonymously. While the sample size is small the research team felt that it was important to complete the paper as a starting point for further research.

\section{Timeframe for Gathering Data}

The data was gathered during a 28-day window in the spring of 2021. The data gathering began on March 30, 2021 and was completed on April 27, 2021.

\section{Types of Analysis Performed}

The analysis examined the survey results to uncover trends in low- and no-code development, including workflow process automation. Inaccurate surveys were eliminated prior to the analysis of the data. This included surveys with incomplete responses. A quantitative approach to data analysis was used. Pivot tables were used to perform an intersectional analysis of the data. The researchers decided against using regression due to the sample size. The tool used to perform the analysis was Microsoft Excel.

First, the researchers used intersectional analysis through an Excel pivot table, mapping the use of low- and no-code development and workflow process automation across various sizes of organizations. Then the researchers used pivot tables to examine the distribution of low- and no-code development tools and the distribution of workflow automation tools across different sizes of organizations. Finally, the researchers used pivot tables to examine the overlap between the usage of low- and no-code tools and workflow automation tools.

\section{Findings}

The survey data indicates the presence of citizen developers using low- and no-code tools in the majority of organizations, as shown in Figure 1. This 
finding is consistent with the research that showed the use of low- and no-code tools is on the rise. Citizen developers are present across the spectrum of all sizes of organizations, as shown in Figure 2. However, this finding is inconsistent with previous preliminary research on low- and no-code development tools which indicated they are primarily used in smaller organizations.

The researchers looked for evidence of where lowcode platforms are in use. Except for small organizations with 1 to 100 employees, respondents in every other size of organization said they have or most likely have citizen developers in their organization, as shown in Figure 3. This is consistent with the research that shows the number of citizen developers is growing, however, this is surprisingly inconsistent with the preliminary research showing low- and no-code development by citizen developers is more popular in small organizations. This finding is surprising since it seems unlikely that large enterprise organizations would relinquish control of technology solutions to employees outside of the IT department.

The survey results also showed that a high percentage of respondents are using workflow automation toolsets, as shown in Figure 4. The use of workflow automation tools seems to be more prevalent in midsize companies than small organizations of 1-100 employees and large organizations of over 5,000 employees. This is not consistent with the existing research, which showed workflow automation tools are more popular in smaller organizations than midsize organizations. However, the finding that workflow automation tools were less popular in the

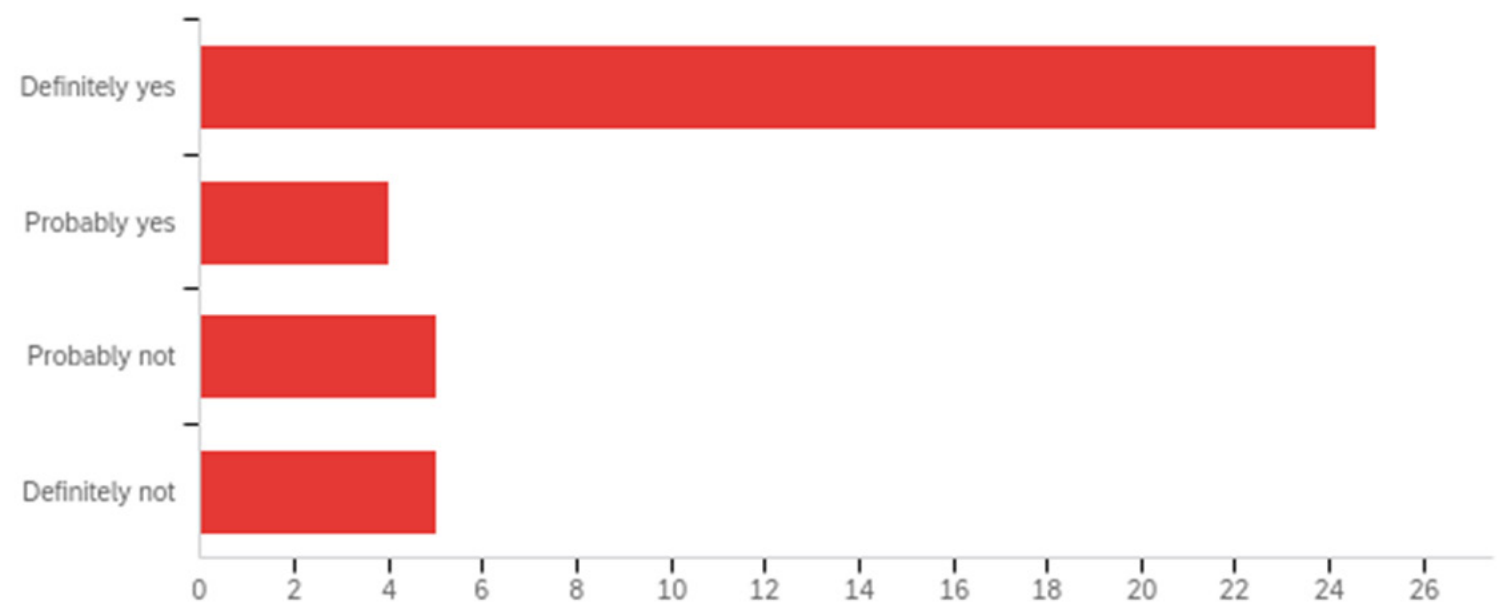

Figure 1. Responses to Survey Question: "Does your organization use a no-code or low-code environment? (Examples: Mendix, PowerApps, Appian)"

\section{Number of Organizations with Citizen Developers by Size}

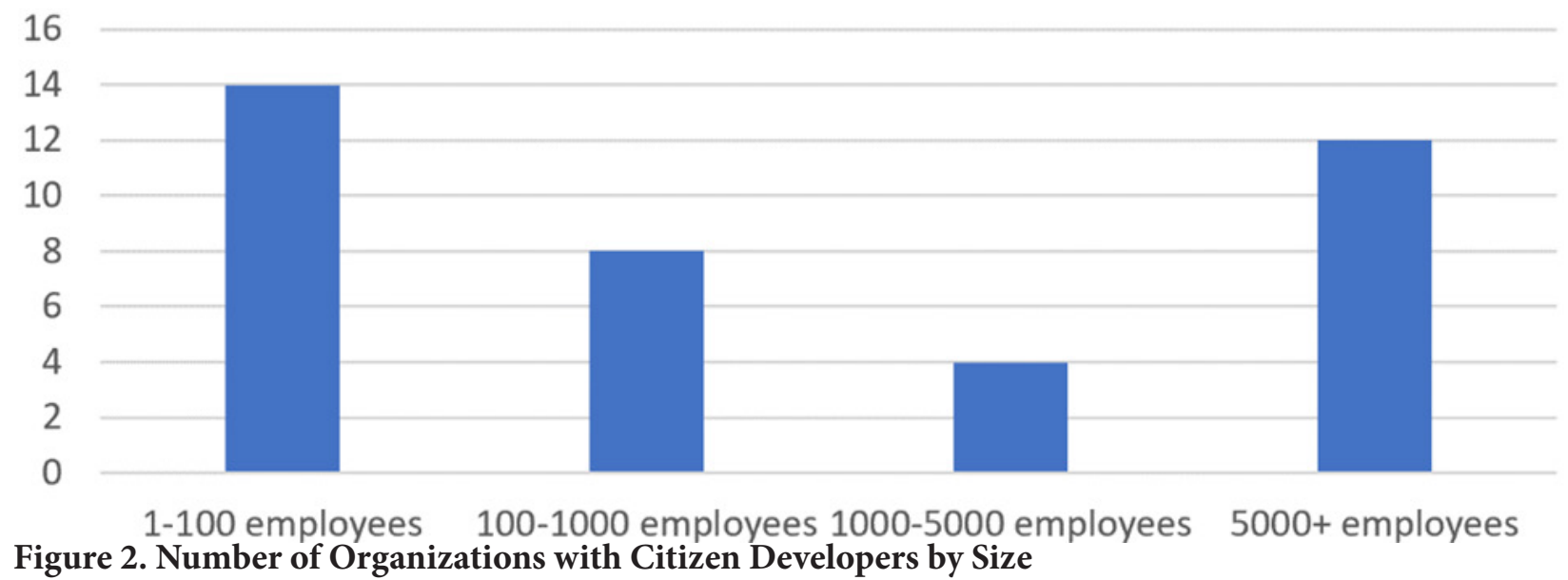

Figure 2. Number of Organizations with Citizen Developers by Size 


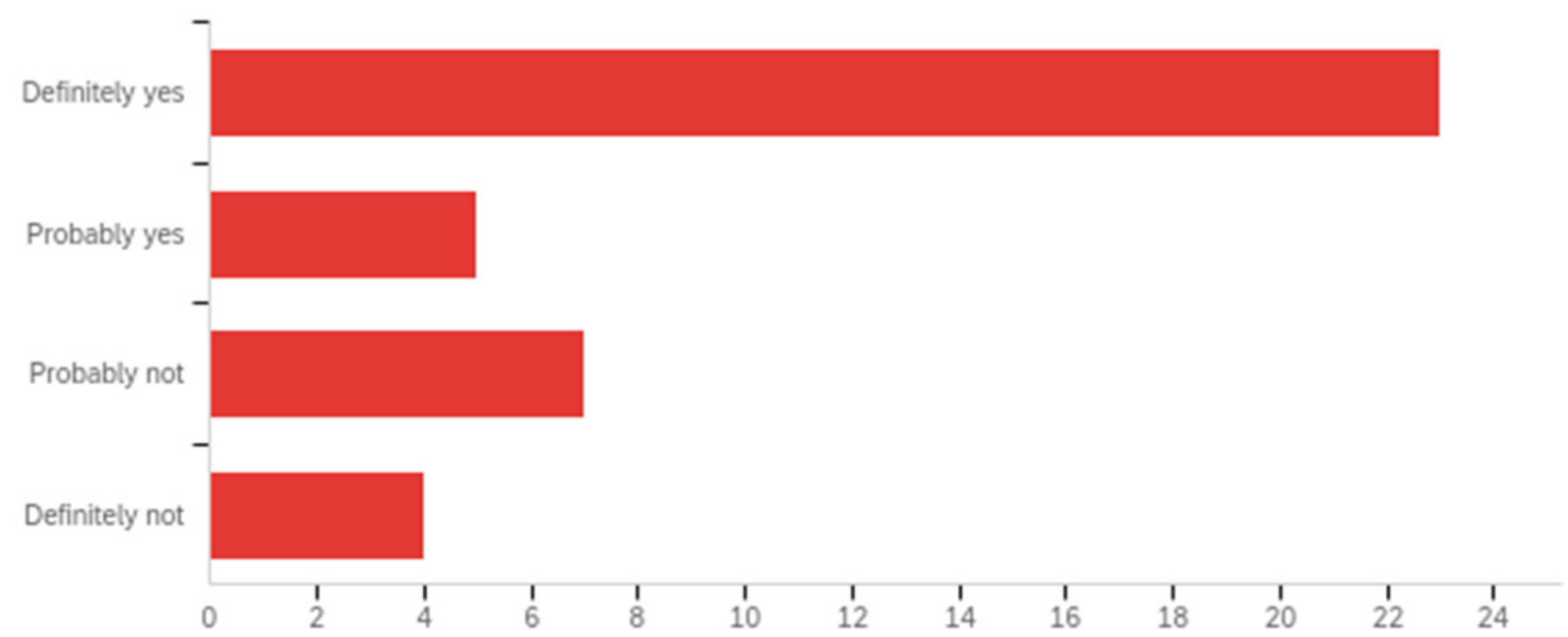

Figure 3. Response to Survey Question: “Do employees outside of your organization's IT department develop technology solutions?"

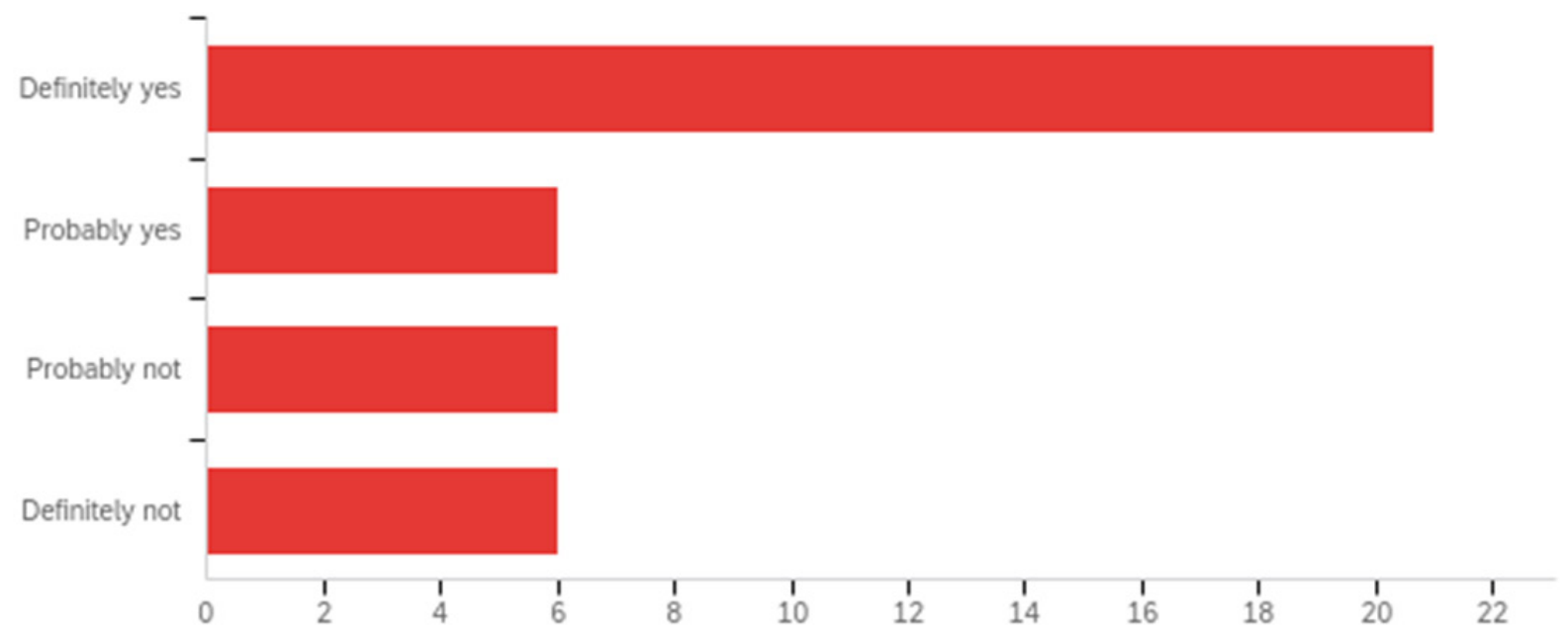

Figure 4. Response to Survey Question: "Does your organization use workflow automation tools? (Examples: Microsoft Power Platform, WorkFusion.)"

largest organizations is consistent with the research which shows these tools are less popular in enterprise organizations.

One of the most interesting findings in the data is that not all of the organizations using low- and nocode development platforms are using workflow automation, while all of the organizations using automation are also using low and no-code development platforms. Figure 5 illustrates this interesting contradiction. This finding is not entirely consistent with the existing research, since the research shows that most organizations use low- and no-code platforms in conjunction with workflow automation, since the toolsets are often sold together to complement one another.

\section{Discussion}

This research was started with the theory that organizations are making greater use of citizen developer tools with low- and no code platforms, based on the literature showing use of these platforms is rising. Since organizations are having difficulty in finding enough software developers to meet their application needs, it makes business sense to use low- and no-code platforms to make up for the shortfall.

The majority of the respondents to the survey indicated that their organization was making use of low- and no-code platforms or is in the process of implementing them within the coming year. This reveals that the answer to RQ1 is that the prevalence of low- and no-code platforms in organizations is high. Low- and no-code platforms are prevalent across all sizes of organizations, which indicates that the answer to RQ2 is that the use of low- and no-code platforms does not differ across organizations of varying sizes. The survey shows that companies of 100 or less employees are making use of these platforms as well as companies with over 5,000 employees. This 


\section{Low-Code and Automation Platform Overlap}
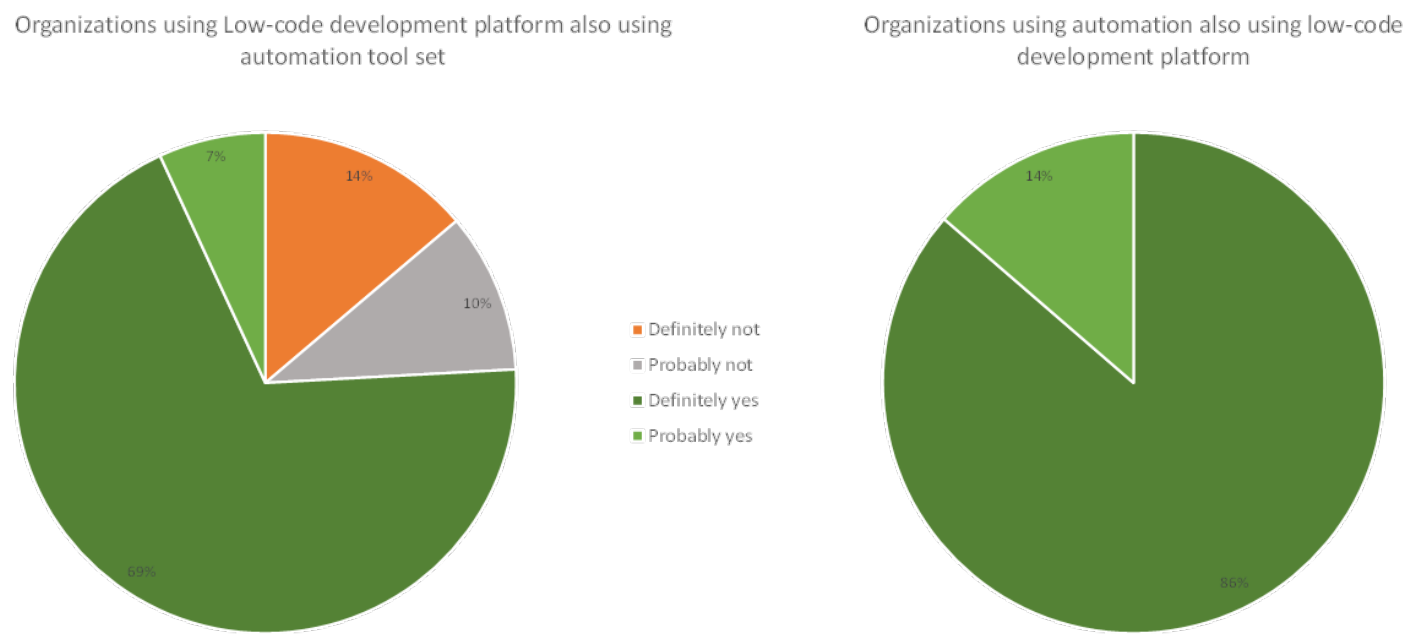

Figure 5. Overlap Between Responses to Survey Questions: "Do employees outside of your organization's IT department develop technology solutions?" and "Does your organization use workflow automation tools?"

indicates that businesses, no matter their size, are turning to the use of low- and no-code platforms, perhaps because they are feeling the resource constraints in finding good developers and are trying to fill in the gaps in their software development teams. This finding is surprising since it seems improbable that larger organizations would allow employees outside of the IT department to develop technology solutions, since larger organizations typically establish an enterprise-wide strategy for technology. Perhaps this points to larger organizations feeling the pinch of the current shortage of software developers, so much so that they are willing to relinquish control of technology to employees outside of the IT department.

The survey results indicate that there is an abundant use of these platforms in small companies of 100 or less employees, which hints at the suggestion that companies can be built around products which are developed using just low- and no-code platforms (Gadberry \& Romero, 2020). This finding is consistent with the 2020 study that found a majority of low- and no-code developers were working in startups or were self-employed (Gadberry \& Romero, 2020). While there needs to be additional research done in this area, it does stir in the imagination the image of a citizen developer entrepreneur creating their own company around an application developed completely on a low- or no-code platform. The growth in the low- and no-code arena increases the technological capabilities for both large and small companies since it makes product development and launch faster and cheaper.
The survey results also show that organizations of all sizes rely on employees outside of the IT department to develop technology solutions. This suggests that the answer to RQ3 is that the prevalence of citizen developers is high. While greater research is needed to quantify the reliance on citizen developers, the results are indicative of the environment in which organizations are finding themselves. Companies must rely on employees outside of their IT department's infrastructure in order to meet their technology needs.

In addition, the results show that use of low- and no-code platforms is prevalent in companies that likewise make use of workflow automation applications, also known as RPA. This indicates the answer to RQ4 is that the relationship between the use of low- and no-code platforms and workflow automation is symbiotic, since most companies who use one also use the other. Since RPA systems are in many cases built into low- or no-code platforms it makes sense that those types of systems work hand-inglove in the companies participating in the survey. However, the finding that the use of low- and nocode platforms does not always overlap with the use of workflow automation is novel. This finding is of particular interest to management since these tools are typically integrated and sold together. It appears some companies are only using a part of the suite of tools.

The need for IT resources, in terms of both people and systems, is rising since digital transformation makes it necessary for organizations to make greater use of transformative technology to evolve (Bahn et 
al., 2016). Digital transformation, coupled with the shortage of development resources, is leading to the rise of the citizen developer armed with low- and no-code platforms (Fryling, 2019). The results of this study indicate that that is the case now and will continue into the future.

\section{Limitations and Further Research}

A key limitation to this research was the sample size, so the research team is planning to repeat this study with additional respondents to form a longitudinal study. Additional research into the link between the shortage of qualified software developers and the prevalence of low- and no-code platforms is warranted. Future research is needed to establish the relationship between the widespread use of lowand no-code platforms and the current shortage of software developers. Furthermore, research could be conducted to see if there is a correlation between low-code and automation and whether the use of one is driving the use of the other in industry. In addition, future research could be done to determine which software vendors are most successful in penetrating the citizen developer's toolbox as well as the reasons that they are. This would provide a deeper insight into the needs of citizen developers.

\section{Conclusions}

From the results of this study, it is evident that citizen developers are real and prevalent across all sizes of organizations. Employees outside of the IT de-

\section{References}

Alamin, M. A. A., Malakar, S., Uddin, G., Afroz, S., Haider, T. B., \& Iqbal, A. (2021). An empirical study of developer discussions on low-code software development challenges. International Journal of Advanced Computer Science and Applications (IJACSA), 1(1), 1-12. https://doi. org/10.1109/MSR52588.2021.00018

Alexander, P. M., Holmner, M., Lotriet, H. H., Matthee, M. C., Pieterse, H. V., Naidoo, S., Twinomurinzi, H., \& Jordaan, D. (2011). Factors Affecting Career Choice: Comparison Between Students from Computer and Other Disciplines. Journal of Science Education and Technology, 20(3), 300-315. https://doi.org/10.1007/s10956010-9254-3

Anderson, H. (2020). Why Low-Code Solutions Are Critical To Optimize Digital Transformation Strategies. KM World, 29(1), 1-4.

Bahn, D., Betz, C., Gluhova, S., Khan, F., Lebens, M., Mosman, M., Paulson, P., Olagunju, A., Opatrny, J., Spencer, G., \& Tarmizi, H. (2016). Renewing the IT curriculum: Responding to agile, DevOps, and digital transformation (Dynamic IT: Digital Transformation Education, pp. 1 - 57). Advance partment are creating solutions to stay competitive and meet their customers' needs. Organizations are utilizing newer technologies such as low- and nocode platforms and workflow automation toolsets to develop technology solutions. The popularity of these solutions is consistent with the existing research. However, the finding that all sizes of organizations are using these tools, not only smaller organizations, is a novel discovery for management since this is inconsistent with the preliminary research. A surprising conclusion is that this rise of the citizen developer is occurring not only in small businesses, as previous research indicated, but across all sizes of organizations. This finding is surprising since it seems less likely that large organizations would cede control of technology solutions to employees outside of the IT department. In addition, the discovery that the use of low- and no-code platforms does not always overlap with the use of workflow automation is also of interest to management since these tools are typically sold together and are often integrated into a single software solution.

The broad implication of this research for the IT field is that the shortage of qualified software developers may be addressed by the rise of the citizen developer, since employees outside of IT are developing technology solutions in all sizes of organizations, not only small startups. The greater impact of this research is that the increasing use of low- and nocode development tools may prove to be a solution to the burgeoning shortage of developers.

IT Minnesota. https://mnstateitcoe.org/wp-content/uploads/2017/10/ReportRenewingITCurriculumOctober19_2017.pdf

Bowman, D. D. (2018). Declining Talent in Computer Related Careers. Journal of Academic Administration in Higher Education, 14(1), 1-4.

Browning, J. (2021, June 17). 7 uses of Robotic Process Automation (RPA) for SMBS: Impact. Impact Networking. Retrieved September 12, 2021, from https://www.impactmybiz.com/blog/blog-7-uses-robotic-process-automation-rpa/.

Casey, K., (2020, July 30). How to explain robotic process automation (RPA) in plain english. The Enterprisers Project. Retrieved September 12, 2021, from https://enterprisersproject.com/ article/2019/5/rpa-robotic-process-automation-how-explain.

Coleman, T. (2020). Democratizing technology: Strategies and tools to empower the government financial professional. The Journal of Government Financial Management, 69(2), 30-35.

Costello, K., \& Rimhol, M. (2021). Gartner forecasts worldwide low-code development technologies market to grow 23\% in 2021. Gartner. https:// www.gartner.com/en/newsroom/press-releas- 
es/2021-02-15-gartner-forecasts-worldwidelow-code-development-technologies-market-togrow-23-percent-in-2021

Duarte, C., \& Ebert, C. (2018). Digital Transformation. IEEE Software, 35(4), 16-21. https://doi. org/10.1109/MS.2018.2801537

Fryling, M. (2019). Low Code App Development. Journal of Computing Sciences in Colleges, 34(6), 119.

Gadberry, A., \& Romero, M. (2020). No-Code Census 2020 (No-Code Census, pp. 1 - 15). Bubble, Inc.

Gartner Information Technology Glossary. (2021). Definition of Citizen Developer. Gartner. https:// www.gartner.com/en/information-technology/ glossary/citizen-developer

Hawkridge, G., Mukherjee, A., McFarlane, D., Tlegenov, Y., Parlikad, A. K., Reyner, N. J., \& Thorne, A. (2021). Monitoring on a shoestring: Low cost solutions for digital manufacturing. Annual Reviews in Control, 51(2), 1-21. https://doi. org/10.1016/j.arcontrol.2021.04.007

Hyun, C. Y. (2019). Design and implementation of a low-code/no-code system. International Journal of Advanced Smart Convergence, 8(4), 188-193. https://doi.org/10.7236/IJASC.2019.8.4.188

Lodge, T., Crabtree, A., \& Brown, A. (2018). IoT app development: Supporting data protection by design and default. Proceedings of the 2018 ACM International Joint Conference and 2018 International Symposium on Pervasive and Ubiquitous Computing and Wearable Computers, (pp. 901 910). https://doi.org/10.1145/3267305.3274151

Martinez, C. \& Pearson, E. (n.d.). The decline of computer science: Two decades of trends. Retrieved September 11, 2021 from https://blog.steppingblocks.com/the-decline-of-computer-science.

Matteson, S. (2020, July 23). Shadow it: The challenges and opportunities. TechRepublic. Retrieved September 11, 2021, from https://www.techrepublic.com/article/the-challenges-and-opportunities-of-shadow-it/.

Moskal, M. (2021). No-code application development on the example of Logotext App Studio platform. Informatyka, Automatyka, Pomiary w Gospodarce i Ochronie Środowiska, 11(1), 54-57. https://doi.org/10.35784/iapgos.2429

Muraski, J., Iverson, J., \& Iversen, K. J. (2021). Building collaboration networks and alliances to solve the IT talent shortage: A revelatory case study. Journal of the Midwest Association for Information Systems, 2021(1), 27-48.

Ngambi, D. (2020). Empathy-driven mobile app development (MAD) without coding: A case of citizen developers. In Critical Mobile Pedagogy (1st ed., pp. 136 - 150). Routledge. https://doi. org/10.4324/9780429261572-10

O'Connor, C. (2021, January 11). RPA: Citizen developers - at the corner of speed and value. CyberArk. Retrieved September 11, 2021, from https:// www.cyberark.com/resources/blog/rpa-citizendevelopers-at-the-corner-of-speed-and-value.

O’Leary, N., \& Conway-Jones, D. (2020). Nodered: Low-code programming for event-driven applications. Accessed: Sept. 15, 2021. [Online]. Available: https://nodered.org/

Olariu, C., Gogan, M., \& Rennung, F. (2016). Switching the Center of Software Development from IT to Business Experts Using Intelligent Business Process Management Suites. In V. E. Balas, L. C. Jain, \& B. Kovačević (Eds.), Soft Computing Applications (pp. 993 - 1,001). Springer International Publishing. https://doi.org/10.1007/978-3-31918416-6_79

Oltrogge, M., Derr, E., Stransky, C., Acar, Y., Fahl, S., Rossow, C., Pellegrino, G., Bugiel, S., \& Backes, M. (2018). The rise of the citizen developer: Assessing the security impact of online app generators. 2018 IEEE Symposium on Security and Privacy (SP), 634-647. https://doi.org/10.1109/ SP.2018.00005

Patterson, S. (2020). Microsoft and salesforce are ranked leaders in competitive low-code space. Software World, 22(November 2020).

Pham, T. (2021, April 13). Council post: Analyzing the software engineer shortage. Forbes. Retrieved September 11, 2021, from https://www. forbes.com/sites/forbestechcouncil/2021/04/13/ analyzing-the-software-engineer-shortage/?sh=791dbdfe321c.

Ploder, C., Bernsteiner, R., Schlögl, S., \& Gschliesser, C. (2019). The future use of LowCode/NoCode platforms by knowledge workers - An acceptance study. In L. Uden, I.-H. Ting, \& J. M. Corchado (Eds.), Knowledge Management in Organizations (pp. 445 - 454). Springer International Publishing. https://doi.org/10.1007/978-3-030-214517_38

Rymer, J. R., \& Koplowitz, R. (2019). The Forrester Wave ${ }^{T M}$ : Low-Code Development Platforms For AD\&D Professionals, Q1 2019.17.

Sahay, A., Indamutsa, A., Di Ruscio, D., \& Pierantonio, A. (2020, August 26). Supporting the understanding and comparison of low-code development platforms. 2020 46th Euromicro Conference on Software Engineering and Advanced Applications (SEAA). https://doi.org/10.1109/ SEAA51224.2020.00036

Sahinaslan, E., Sahinaslan, O., \& Sabancioglu, M. 
(2021). Low-code application platform in meeting increasing software demands quickly: SetXRM. AIP Conference Proceedings 2334, 070007. https://doi.org/10.1063/5.0042213

Sakhnyuk, P. A., \& Sakhnyuk, T. I. (2020). Intellectual technologies in digital transformation. IOP Conference Series: Materials Science and Engineering, 873, 12-16. https://doi.org/10.1088/1757899X/873/1/012016

Sayer, P. (2020, December 11). SAP rolls out RPA, low-code tools to help cut dev debt. CIO. https:// www.cio.com/article/3601053/sap-rolls-out-rpalow-code-tools-to-help-cut-dev-debt.html

Shafagatova, A., \& Van Looy, A. (2021). Developing a tool for process-oriented appraisals and rewards: Design science research. Journal of Software: Evolution and Process, 33(3), e2321. https:// doi.org/10.1002/smr.2321

Silva, C., Vieira, J., Campos, J. C., Couto, R., \& Ribeiro, A. N. (2020). Development and validation of a descriptive cognitive model for predicting usability issues in a low-code development platform. Human Factors: The Journal of the Human Factors and Ergonomics Society, 1-21. https://doi. org/10.1177/0018720820920429

Steinmetz, D. (2021, March 9). Get started with Power Automate. Microsoft Documentation: Power Automate. https://docs.microsoft.com/en-us/ power-automate/getting-started

Tariq, H. (2021, May 7). Low-code versus no-code and the future of application development. Forbes. https://www.forbes.com/sites/forbescommunicationscouncil/2021/05/07/low-code-versus-no-code-and-the-future-of-application-development/

Totterdale, R. L. (2018). Case study: The utilization of low-code development technology to support research data collection. Issues In Information Systems, 19(2), 132-139. https://doi.org/10.48009/2_ iis_2018_132-139

Tuomi, K. (2018). The technology trade-off: The cost of software development and its implications for start-ups (SSRN Scholarly Paper ID 2978181). Social Science Research Network. https://doi. org/10.2139/ssrn.2978181

Van der Aalst, W. M. P., Bichler, M., \& Heinzl, A. (2018). Robotic process automation. Business \& Information Systems Engineering, 60(4), 269-272. https://doi.org/10.1007/s12599-018-0542-4

Van Looy, A. (2020). Innovating organizational processes with new technologies: Problems and solutions. IT Professional, 22(5), 71-80. https://doi. org/10.1109/MITP.2020.2969614

Vial, G. (2019). Understanding digital transformation: A review and a research agenda. The Journal of Strategic Information Systems, 28(2), 118-144. https://doi.org/10.1016/j.jsis.2019.01.003

Vincent, A. P., Natis, Y., Iijima, K., Wong, J., Ray, S., \& Jain, A. (2020). Magic quadrant for enterprise low-code application platforms (Magic Quadrant, pp. 1 - 32). Gartner.

Wild, J. (2021). Commentary: Beyond data: The mindsets and disciplines needed to fuel growth. Journal of Marketing, 85(1), (pp. 190 - 195). https://doi.org/10.1177/0022242920972398

Wolff, I. (2019). Software: Making in-house apps with low-code, no-code platforms. Manufacturing Engineering, 163(4), (pp. 58 - 67).

Woo, M. (2020). The rise of no/low code software development-no experience needed? Engineering (Beijing, China), 6(9), (pp. 960 - 961). https:// doi.org/10.1016/j.eng.2020.07.007 


\section{Authors}



